\title{
Leptonic Decays of Heavy-Light Systems
}

\author{
Chris Allton ${ }^{\mathrm{a} *}$ \\ aDipartimento di Fisica, Università di Roma "La Sapienza", and INFN, Sezione di Roma I, \\ P.le Aldo Moro, 00185 Roma.
}

Results from recent lattice calculations of the decay constants $f_{B}$ and $f_{D}$ are reviewed. A discussion of the methods currently used is presented, together with an outline of the various systematic effects involved.

\section{Introduction}

Weak matrix elements of heavy-light mesons are of fundamental importance in particle physics since they enter determinations of some of the least known CKM matrix elements, and of the $B-\bar{B}$ and $D-\bar{D}$ mass splittings. The simplest matrix element to study is the leptonic decay constant of a pseudoscalar meson, $f_{P}$. It is defined:

$<0\left|A_{\mu}\right| P>=f_{P} p_{\mu}$,

where $\mid P>$ is a pseudoscalar meson with 4momentum $p_{\mu}$, and $A_{\mu}$ is the axial current.

The feasibility of using the lattice technique to calculate $f_{P}$ is now firmly established. The general trend in lattice calculations of $f_{P}$ is now towards a greater understanding of the systematic effects entering the calculation. This has been made possible through smaller statistical errors uncovering systematics which were previously hidden. Thus, the effects of, e.g., quenching, different choices of interpolating operators etc., can be studied. Systematic effects will be a focus throughout much of this review.

The plan of this review is as follows. The next section overviews the three methods currently employed by the lattice community to calculate $f_{P}$. Recent results from each of these methods are then presented in the subsequent sections. The present status of lattice calculations is summarised in the conclusion.

Unless explicitly stated, all results are to be taken as "preliminary".

\footnotetext{
*Address from 1st October, 1995: Department of Physics, University of Wales, Swansea, Singleton Park, Swansea SA2 8PP, U.K. Preprint Rome 95/1114 \& SWAT/90
}

\section{Overview of Methods Used}

Heavy Quark Effective Theories (HQET) (see e.g. [1]) are invaluable tools in the study of the spectrum and decays of systems involving one or more heavy quarks. In this theory, the QCD action is systematically expanded in terms of the inverse heavy quark mass, $m_{Q}$.

HQET is extremely powerful in lattice calculations as well as in the continuum. It provides a means of overcoming the problem of simulating quarks with mass greater than the inverse lattice spacing. In general, a lattice calculation using HQET proceeds much as in conventional lattice calculations, but with all heavy quark propagators calculated using HQET. Thus a heavy-light pseudoscalar meson correlation function in the conventional approach would be, e.g.

$$
\begin{aligned}
C(t) & =\sum_{\vec{x}}<A_{0}(x) A_{0}(0)> \\
& =\sum_{\vec{x}} \operatorname{Tr}\left\{S_{q}(x, 0) \gamma_{5} S_{Q}(0, x) \gamma_{5}\right\}
\end{aligned}
$$

where $S_{q}$ is the light quark propagator calculated using the traditional discretised Dirac equation, and the heavy quark propagator, $S_{Q}$, is calculated using the discretised HQET action up to a chosen order in $1 / m_{Q}$.

In this review I will be discussing results obtained with $S_{Q}$ calculated using

- the zeroth order HQET (also termed the 'static' case)

- HQET to some finite (typically the first) order (also termed NRQCD). 


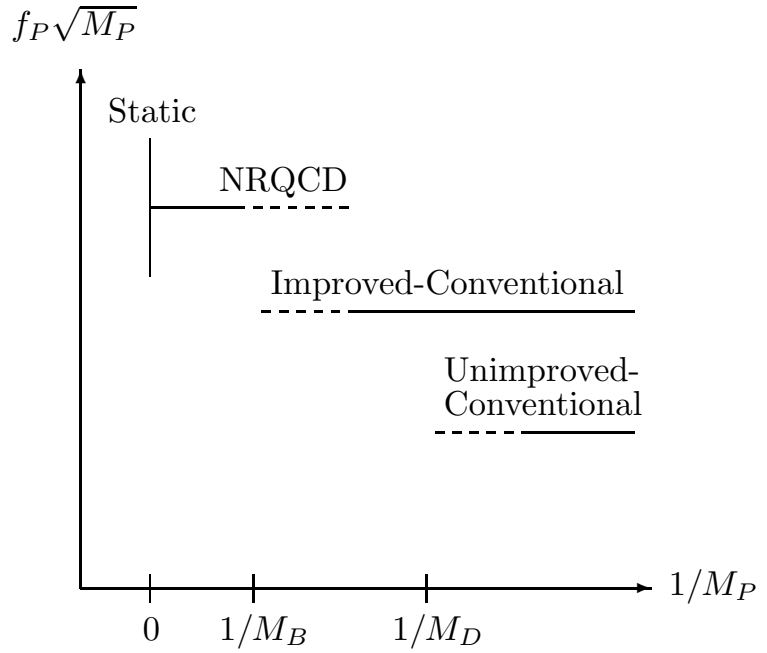

Figure 1. Representation of the range of validity of the three methods discussed in this review.

I will also discuss results with $S_{Q}$ determined from

- the conventional lattice Dirac equation (which I will term the "conventional" approach).

The next three sections discuss recent results from each of the above approaches in turn. The lattice actions or $S_{Q}$ 's used in each case are defined in the corresponding sections.

Some general comments regarding the applicability of the three approaches are helpful at this stage. One can represent the range of validity of each of the above methods graphically in fig.1. Here $f_{P} \sqrt{M_{P}}$ is plotted against $M_{P}$. Note that in HQET it can be shown that, up to unimportant logarithms,

$f_{P} \sqrt{M_{P}}=$ constant $+O\left(1 / M_{P}\right)$.

The static method is valid only for $m_{Q}=\infty$ and serves as a reference point for extrapolations in $1 / m_{Q}$. The NRQCD approach [2,3] is valid for $1 /\left(m_{Q} a\right)<<1$, and, at $O\left(1 / m_{Q}\right)$ its range of validity should include masses down to around $M_{B}$. The conventional approach, for present lattice parameter values, is believed to be accurate for meson masses of around $M_{D}$ and less. In fact, this statement assumes that some improve-

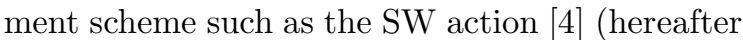
termed the "Clover" action) and/or the "Fermilab" (also termed the "Heavy") formalism [5] is implemented. If no such improvement is undertaken, then the conventional approach fails for $M_{P} \gtrsim M_{D}$.

Taken together, these three approaches span the entire range of $m_{Q}$, from infinity to the charm quark mass, $m_{c}$ and below. Clearly, a necessary condition for the success of lattice calculations is that these three methods agree in the shared regions, and that they provide a continuous functional behaviour for the quantities measured. It is one of the purposes of this review to study the status of this consistency check.

To set the notation, lattice calculations of $f_{P}$ determine the dimensionless, unrenormalised quantity $\phi^{\#}$ corresponding to $f_{P}$. It is defined (using an obvious notation):

$f_{P} \sqrt{M_{P}}=Z^{R e n} \phi^{\#} a^{-3 / 2}$.

(Note other notation for $\phi^{\#}$ appearing in the literature includes $\sqrt{2} Z_{L}$ and $\sqrt{2} \tilde{f_{B}}$.) I have chosen the superscript ${ }^{\#}$ to denote unrenormalised, dimensionless lattice quantities.

\section{Static Results}

The static action has a very simple form and allows the heavy quark to propagate only in time, and not in position space. This action can be inverted to give the following form for $S_{Q}$ [9, 10]:

$S_{Q}(x, 0)=\mathcal{P}_{\vec{x}}\left(t_{x}\right) \delta(\vec{x}) \theta\left(t_{x}\right) e^{-m_{Q} t_{x}} \frac{1+\gamma_{4}}{2}$

where $\mathcal{P}_{\vec{x}}\left(t_{x}\right)$ is the Polyakov line from the point $(\vec{x}, 0)$ to $\left(\vec{x}, t_{x}\right)$.

\subsection{Smearing}

The history of the static approach on the lattice has been plagued by problems associated with its poor signal to noise ratio. The reason for this effect is now well understood in terms of the variance of the correlation function picture [11]. An approach to circumvent this problem is to intro- 
duce smeared interpolating operators [12]. These can be expressed as

$A_{0}^{S}(\vec{x}, t)=\sum_{\vec{y}, \vec{z}} \bar{Q}(\vec{y}, t) \gamma_{0} \gamma_{5} q(\vec{z}, t) \psi(|\vec{x}-\vec{y}|,|\vec{x}-\vec{z}|)$.

$A_{0}^{S}$ still couples to the ground state because it has the same quantum numbers as the local current $A_{0}^{L}$, but using it to define correlation functions can result in a smaller overlap with the excited states.

There is a wide variety of forms of the smearing function $\psi$ presently in use. The most simple form of smearing functions is 'cubic' defined [13]:

$$
\begin{aligned}
\psi(\vec{y}, \vec{z})= & \delta(\vec{z}) ; \\
& \text { for all }\left|y_{i}\right| \leq L_{s} / 2 ; \mathrm{i}=1,2,3 \\
= & 0 \text { otherwise. }
\end{aligned}
$$

These have the advantage that they are easiest to code, especially on parallel machines, but have the disadvantage that they require a gauge fixing procedure and are a rather brutal approximation to the physical wave function. This second point will be discussed more in sec 3.5 .

Another popular choice of smearing functions is based on the non-relativistic quark model [14]. Here, the heavy quark potential of the gauge configurations in the simulation is used to obtain the quantum mechanical wave function. Its advantage is that the physics is being used to dictate the smearing.

The Wuppertal collaboration have proposed various smearings [15, 16]. The advantage in these cases is that the smearing is relatively quick, and is gauge invariant.

My favourite smearing is "MOST" (Maximal Operator Smearing Technique) [17. In this technique the set of operators is defined where the $Q$ and $q$ fields have every possible separation (which is independent under cubic symmetry). The twopoint correlation between all members of this set is measured, and an simple analysis [18, 19] can be used to extract the ground state properties. The advantage of this method is obvious: because it uses all possible relative separations as a basis, all other smearing methods are a subset of this approach. Its use in light-light physics should there- fore be investigated. The disadvantage is that it becomes memory intensive for large lattices.

Note that in order to extract the local matrix element, $f_{P}$, local-smeared, as well as smearedsmeared correlation functions need to be measured. In practice it is best to place the local current at the sink rather than the source, since a better signal to noise ratio results [20,16].

\section{2. $Z^{\text {Ren }}$}

In order to define the physical $f_{P}$ a choice needs to be made for the definition of the renormalisation constant $Z^{\text {Ren }}$ see eq.(11). Perturbative calculations 21] show, in the case of the staticWilson current,

$Z_{\text {stat }}^{\text {Ren }}\left(g^{2}\right)=\left(1-.189 g^{2}\right)\left(1+.0127 g^{2} \log \left(a^{2} m_{Q}^{2}\right)\right)$.

For the static-Clover case, 0.189 is replaced by 0.144 [22]. Note that the first factor is essentially the lattice to continuum matching within the effective (static) theory, and the second is the matching between the effective and full theory in the continuum.

Using the Kronfeld-Lepage-Mackenzie formalism [23,7] leads to the following definition of $Z_{\text {stat }}^{R e n}$ for the Wilson action,

$$
\begin{aligned}
Z_{\text {stat }}^{\text {Ren }}\left(g^{2}\right)= & \frac{\sqrt{1-6 \tilde{\kappa_{q}}}}{\sqrt{2 \kappa_{q}}} \times\left(1-0.137 \tilde{g}^{2}\right) \\
& \times\left(1+0.0127 \tilde{g}^{2} \log \left(a^{2} m_{Q}^{2}\right)\right) .
\end{aligned}
$$

Here, the renormalised couplings are $\tilde{g}^{2}=6 / \beta / u_{0}^{4}$ and $\tilde{\kappa_{q}}=u_{0} \kappa_{q} . \quad u_{0}$ will be defined later. The first factor in eq.(1) is the rescaling of the light quark field from the traditional, Wilson $\sqrt{2 \kappa_{q}}$ to the KLM prescription. There is however an ambiguity in the above definition of $Z_{\text {stat }}^{R e n}$. This is because one is free to define $u_{0}$ as, e.g. the average plaquette, $\left\langle U_{\text {Plaq }}\right\rangle^{1 / 4}$, or $1 / 8 \kappa_{c}$ (where $\kappa_{c}$ is the critical value of the hopping parameter).

Table 1 displays the values of $Z_{\text {stat }}^{R e n}$ obtained with the above definitions of $Z_{\text {stat }}^{R e n}$. As can be seen from the table the difference between the various determinations of $Z^{\text {Ren }}$ is $O(30 \%)$ and it is $O(5 \%)$ even within the KLM formalism. Obviously, this means that the overall systematic error in $f_{B}^{s t a t}$ has a contribution of this order from the uncertainties in $Z^{R e n}$. This error from the 
Table 1

$Z_{\text {stat }}^{R e n}$ obtained using different definitions. Column 2 uses the "naive" definition of $g^{2}=6 / \beta$ (see eq.(30). Columns 3 and 4 use the KLM formulation (at the chiral limit) with the $u_{0}$ definitions given by $1 /\left(8 \kappa_{c}\right)$ and $<U_{\text {plaq }}>^{1 / 4}$ respectively (see eq.(位).

\begin{tabular}{lccc}
\hline$\beta$ & \multicolumn{3}{c}{$Z_{\text {stat }}^{\text {Ren }}$} \\
\hline 6.0 & 0.83 & 0.62 & 0.59 \\
6.1 & 0.83 & 0.64 & 0.61 \\
6.2 & 0.83 & 0.66 & 0.63 \\
6.3 & 0.83 & 0.67 & 0.64 \\
6.4 & 0.83 & 0.68 & 0.65 \\
\hline
\end{tabular}

determination of $Z_{\text {stat }}^{R e n}$ has now become one of the dominant errors in the lattice calculations of $f_{B}^{s t a t}$. The way around this problem is to use a non-perturbative definition of $Z^{R e n}$ which are becoming available which circumvent the above problems completely [24].

\subsection{Continuum Limit}

The possible variation of $f_{P}$ in the static limit with the lattice spacing, $a$, has been long discussed (see, for example, 25, 16, 14 This so-called non-scaling behaviour has been proposed as a mechanism for reducing the lattice prediction for $f_{P}$ by as much as $30 \%$. In principle this $a$ dependence is simple to study. One simply calculates $f_{P}$ on the lattice using a number of $\beta$ values and extrapolates the results to $a=0$. In practice this method is difficult to implement due to the increasing statistical errors of the data points closest to the point of interest, $a=0$.

Recently a method has been proposed to analyse the scaling behaviour of $f_{P}$, and indeed any other dimensionful lattice quantity [26]. Instead of studying the scaling of the final quantity, $f_{P}$, the scaling of each of the three factors in eq.(1) which define $f_{P}: Z_{\text {stat }}^{R e n} ; \phi^{\#}$; and $a^{-1}$ are determined separately. Each of these three factors are functions of $g^{2}$. The question to be answered is: does the $g^{2}$ dependence of these factors cancel in the overall product? The analysis performed in [26] suggests that, within present statistical er- rors, $f_{P}$ does scale but only for $\beta \gtrsim 6.0$. This means that analyses which use a linear fit in $a$ and data with $\beta \lesssim 6.0$ are biasing their continuum extrapolation to a smaller value. This is due to the levering effect of the non-scaling, and typically higher data for $\beta \lesssim 6.0$, which, furthermore, typically have smaller statistical errors.

The analysis in 26] also showed that the dominant non-scaling effect was not due to higher orders in perturbation theory in $g^{2}=6 / \beta$, but rather to $O(a)$ effects. A common $20 \%$ to $30 \%$ discretisation effect was found for the quantities studied, such as $\phi^{\#}, M_{\rho}$ and $f_{\pi}$.

\subsection{Recent Results}

Recent results for $f_{B}^{\text {stat }}$ are shown in table 2 together with their references in the second row. A striking fact about these latest simulations is the appearance of simulations with dynamical fermions [27]28]. A discussion of the effects of this on $f_{P}$ appears in secs. 4.2,5.1 and in the conclusion.

A brief description of each of the groups' work, together with a longer discussion on the results from the APE collaboration follows. I have chosen to present a relatively detailed discussion of the APE collaboration's work since it will not be presented elsewhere in the proceedings.

The UKQCD Collaboration [29] presented results for both $f_{B}^{\text {stat }}$ and $B_{B}^{\text {stat }}$ (see also [30]). They noted that the systematic error associated with $Z^{\text {Ren }}$ is now a major uncertainty (see sec.3.2). A summary of their results is shown in table 2 . (The first error is statistical and the second systematic.)

The SGO collaboration 31,27 used both the static and NRQCD implementation of the heavy quark propagator, and their results will be discussed in detail in the NRQCD section 4 .

The MILC collaboration [28] combine an analysis of both the static and conventional approaches, and a discussion of their work will appear in the latter section (sec. 5.1).

\subsection{APE Results}

The APE collaboration has recently carried out a high statistics calculation of $f_{B}^{\text {stat }}$ in the quenched approximation [32]. This work is a con- 
Table 2

Summary of recent results using the static approach. 'Q' signifies Quenched simulation, 'D' Dynamical. See text for detailed comments. All numbers should be considered preliminary with the exception of [30].

\begin{tabular}{|c|c|c|c|c|}
\hline $\begin{array}{l}\text { Collaboration } \\
\text { Reference }\end{array}$ & $\begin{array}{c}\text { UKQCD } \\
29,30\end{array}$ & $\begin{array}{l}\text { SGO } \\
27,31\end{array}$ & $\begin{array}{c}\text { MILC } \\
28\end{array}$ & $\begin{array}{c}\text { APE } \\
\text { [32 \& see text }\end{array}$ \\
\hline \multicolumn{5}{|c|}{ Lattice Parameters } \\
\hline$n_{F}$ & 0 & $0 \& 2$ & $0 \& 2$ & 0 \\
\hline$\beta$ & 6.2 & $\begin{array}{l}5.6 \mathrm{D} \\
6.0 \mathrm{Q}\end{array}$ & $\begin{array}{l}5.7 \rightarrow 6.5 \mathrm{Q} \\
5.4 \rightarrow 5.7 \mathrm{D}\end{array}$ & $6.0,6.1,6.2,6.4$ \\
\hline Volume & $24^{3} \times 48$ & & $\leq 32^{3} \times 100$ & $\leq 24^{3} \times 64$ \\
\hline$N_{c f g s}$ & 60 & $\begin{array}{c}100 \mathrm{D} \\
35 \mathrm{Q}\end{array}$ & $O(100)$ & $\lesssim 400$ \\
\hline $\begin{array}{l}S_{q} \\
S_{Q}\end{array}$ & $\begin{array}{l}\text { Clover } \\
\text { Static }\end{array}$ & $\begin{array}{c}\text { Wilson \& Clover } \\
\text { Static } \\
\text { \& NRQCD } \\
\end{array}$ & $\begin{array}{c}\text { Wilson } \\
\text { Static } \\
\text { \& Conventional } \\
\end{array}$ & $\begin{array}{c}\text { Wilson \& Clover } \\
\text { Static }\end{array}$ \\
\hline \multicolumn{5}{|l|}{$\overline{\text { Results }}$} \\
\hline $\begin{array}{l}f_{B}^{\text {stat }}[\mathrm{MeV}] \\
f_{B_{S}}^{\text {stat }} / f_{B}^{\text {stat }} \\
M_{B_{S}}-M_{B}[\mathrm{Me}\end{array}$ & $\begin{array}{c}266_{-20-27}^{+18+28} \\
1.16_{-3}^{+4} \\
87_{-12-12}^{+15+6} \\
\end{array}$ & $\begin{array}{c}\text { see Section } 4 \\
" \\
"\end{array}$ & $\begin{array}{c}\text { see Section } 5 \\
" \\
"\end{array}$ & $\begin{array}{l}240(30) \\
1.17(4) \\
81(10) \\
\end{array}$ \\
\hline
\end{tabular}

tinuation of a long program of work in this area 33 35. As discussed in sec. 3.1, the cubic smearing function was used. The methods used in the past for extracting the local matrix element $\phi^{\#}$ from the smeared correlation functions are discussed in [34]. One problem with these methods is that they require the selection of the "best" cube size $L_{s}$ (see eq.(2)). A number of techniques were developed to make this choice as free from bias as possible [34,35]. However, the problem remained that cubes are available at only discrete sizes, and that as the light quark mass was varied, the meson's variation in size could not be adequately tracked by the cubes. For example, at $\beta=6.4$ the best cube size for the heaviest of the light quarks studied was clearly $L_{s}=15$, but for the lightest quark was somewhere between $L_{s}=15$ and $L_{s}=17$.

A solution to this problem has already been proposed by a number of authors (see e.g. [14): one includes various cube sizes altogether in the one $\chi^{2}$ fit so that the fit is not constrained by a single cube size. Thus, in the example considered above for $\beta=6.4$, the new enlarged $\chi^{2}$ is

$$
\chi^{2}=\sum_{L_{S}=15,17} \sum_{t} \sum_{i=S S, L S}\left(\frac{C^{L_{S}, i}(t)-f^{L_{S}, i}(t)}{\sigma^{L_{S}, i}(t)}\right)^{2} .
$$

In this definition, both the $L_{s}=15$ and $L_{s}=17$ cubes are included in the $\chi^{2}$ as well as the usual sum over the times $t$ in the fitting window. The sum $i=S S, L S$ includes the contributions from the smeared-smeared and the smeared(at source)local(at sink) correlation functions. The fitting function $f^{L_{S}, i}(t)$ is chosen appropriately; for example,

$$
\begin{aligned}
f^{L_{S}=15, i=L S}(t) \equiv & \frac{\phi^{\#} \phi^{\#}(15)}{2} e^{-M t} \times \\
& \left(1+\eta(L) \eta(15) e^{-\Delta t}\right)
\end{aligned}
$$

where a two state fit is performed and the $\eta$ 's and $\Delta$ are the overlap and mass gap of the first excited state respectively.

The APE Collaboration has re-done its analysis for its whole data set at $\beta=6.0,6.1,6.2$ and 6.4 using the above method. As an example, fig. 2 shows the results of the new method together with the previous analysis in the case of $\beta=6.4$. As can be seen, the use of the new method results in an increased slope in the chiral extrapolation. Thus the chiral ratios and differences, $f_{B_{S}}^{\text {stat }} / f_{B}^{\text {stat }}$ and $M_{B_{S}}-M_{B}$ will increase. The overall effect is not large, but, because the statistical errors are getting smaller, systematic effects such as this are becoming important. 


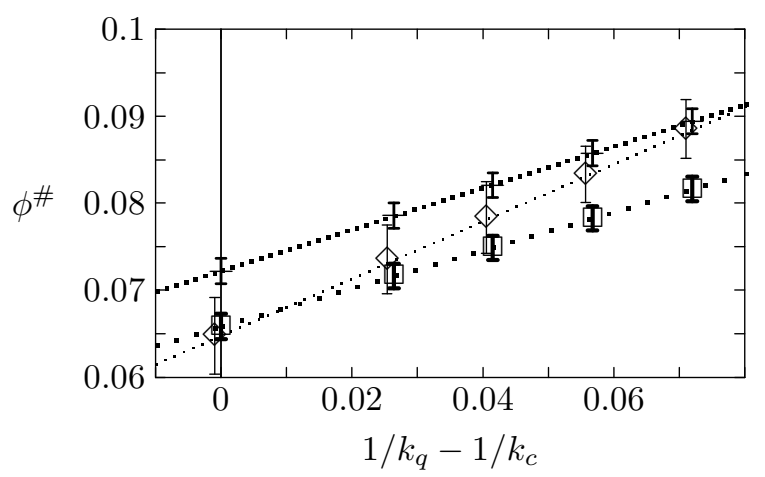

Figure 2. APE results for $\phi^{\#}$ as a function of the quark mass $1 / \kappa_{q}-1 / \kappa_{c}$. The data shown is for $\beta=6.4$ with the Clover action for the light quarks. The plot shows the results of the old method for $L_{s}=15$ ("+") and 17 (" $\square$ "), and the new method ("ऽ") (see text). Points from the new method have been shifted horizontally to aid clarity.

In fig. 3 , the values for $f_{B}^{\text {stat }}$ at various $\beta$ values are plotted against the lattice spacing $a$ using the new method. In this plot, the rho mass was used to set the scale. A preliminary fit to a constant (see sec.3.3) results in $f_{B}^{\text {stat }}=240(30) \mathrm{MeV}$. Values of $f_{B_{S}}^{\text {stat }} / f_{B}^{\text {stat }}=1.17(4)$, and $M_{B_{S}}-M_{B}=$ 81(10) MeV (compared to 96(6) Mev from experiment) are also predicted. All errors quoted here include both the statistical and $a \rightarrow 0$ uncertainties. Finite volume effects are believed to be negligible for these lattices [16].

\section{NRQCD Results}

The NRQCD action used in current simulations is correct to $O\left(1 / m_{Q}\right)$ [2, 3]:

$\mathcal{L}=Q^{\dagger}\left\{-D_{t}+\frac{\boldsymbol{D}^{2}}{2 m_{Q}}+\frac{g}{2 m_{Q}} \sigma \cdot \boldsymbol{B}\right\} Q$.

This action can be simply inverted to obtain the heavy quark propagator. In addition to the $1 / m_{Q}$ terms which appear in the action, there are $1 / m_{Q}$ terms which appear in the definition of the currents [36]. These must be included in order to make the calculation correct at this order. All

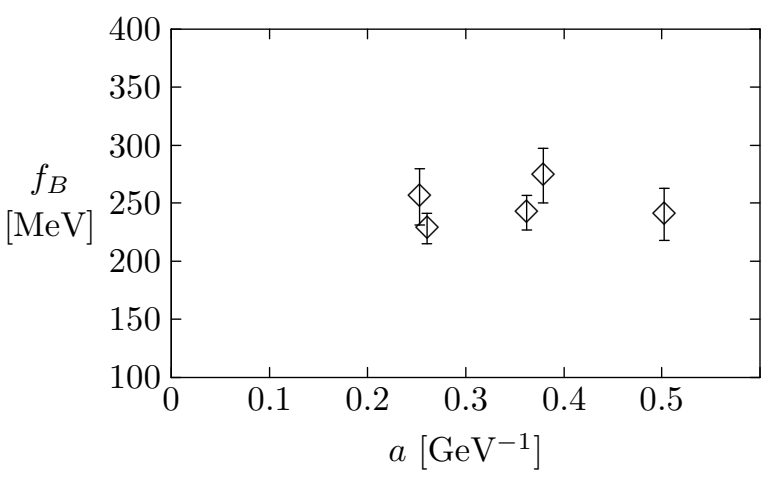

Figure 3. APE results for $f_{B}^{\text {stat }}$ for the various simulations as a function of the lattice spacing $a$ (set from the rho mass). From right to left the data is for Clover $\beta=6.0$, Wilson $\beta=6.1$, Clover $\beta=6.2$, Wilson $\beta=6.4$ and Clover $\beta=6.4$. Horizontal errors are suppressed for clarity.

the NRQCD work presented at this conference took these terms into account.

The NRQCD approach is valid for heavy-light meson masses down to around the B-meson. Unfortunately it is not valid at the charm mass where higher order terms in the $1 / m_{Q} a$ expansion become relevant [37]. In theory, moving to a larger $a$ value (smaller $\beta$ ) would mean that these terms become less relevant. In practice however, this would only increase the $O\left(m_{q} a\right)$ effects to an extent where the results would be unreliable in any case.

Table 3 summarises the data presented in this conference with the references appearing in the second row. The following sections detail the results of the SGO (SCRI- Glasgow-Ohio) collaboration. In the first, the quenched approximation was used, and in the second dynamical quarks were included. See [38] for details of the Kentucky calculation.

\subsection{SGO Results: Quenched}

The SGO collaboration's quenched data 31] are run using the Clover action for the light quarks: both the tadpole unimproved and improved (i.e. $c=1$ and $\sim 1.4$ respectively, where $c$ is the coefficient of the Clover term in the ac- 
Table 3

Summary of recent results using the NRQCD approach. See text for detailed comments. All numbers should be considered preliminary.

\begin{tabular}{|c|c|c|c|}
\hline $\begin{array}{l}\text { Collaboration } \\
\text { Reference }\end{array}$ & $\begin{array}{r}\text { SGO } \\
31]\end{array}$ & $\begin{array}{l}\text { SGO } \\
{[27]}\end{array}$ & $\begin{array}{c}\text { Kentucky } \\
38\end{array}$ \\
\hline \multicolumn{4}{|c|}{ Lattice Parameters } \\
\hline$n_{F}$ & 0 & 2 & 0 \\
\hline$\beta$ & 6.0 & 5.6 & 6.0 \\
\hline Volume & $16^{3} \times 48$ & $16^{3} \times 32$ & $20^{3} \times 30$ \\
\hline$N_{c f g s}$ & $\sim 50$ & 100 & 32 \\
\hline$S_{q}$ & Clover & Wilson \& Clover & Wilson \\
\hline \multirow[t]{2}{*}{$S_{Q}$} & NRQCD to $O\left(1 / m_{Q}\right)$ & NRQCD to $O\left(1 / m_{Q}\right)$ & NRQCD to $O\left(1 / m_{Q}\right)$ \\
\hline & \& Static & \& Static & \\
\hline \multicolumn{4}{|l|}{$\overline{\text { Results }}$} \\
\hline$f_{B}[\mathrm{MeV}]$ & $\sim 160(40)$ & $\sim 190$ & \\
\hline \multirow[t]{2}{*}{$f_{B_{S}}^{\text {stat }} / f_{B}^{\text {stat }}$} & & 1.18(3) Clover & $1.15_{-1}^{+2}$ \\
\hline & & 1.28(4) Wilson & \\
\hline
\end{tabular}

tion). Two values of $\kappa_{q}$ were used which straddle the mass of the strange quark. Four values of the NRQCD quark mass were used. The results for $\phi^{\#}$ at the chiral limit versus $1 / M_{P}^{\#}$ are plotted in fig. 4 for the $c=1$ case together with the static point. Fitting only the NRQCD points shows that they can be made to extrapolate to the static point only if a quadratic term, $c_{2} / M_{P}^{2}$, is included in the fit. If a linear term only is allowed, then the static point seems too high. However, it may be that with the inclusion of more $m_{Q}$ values closer to the static point, this apparent discrepancy disappears (see sec.4.2). Also, the $c=1.4$ data (not shown) has a better agreement between the NRQCD data and the static point [31].

A preliminary calculation of the renormalisation constant relevant for the NRQCD-light axial current has been performed [39]. Using this value, the SGO collaboration obtains $f_{P}$ at their 4 values of $m_{Q}$. From these, I obtain the preliminary estimate of $f_{B}$ which appears in table 3 .

\subsection{SGO Results: Dynamical}

Dynamical simulations involving NRQCD were presented by the SGO Collaboration in last year's conference [40]. This year's results [27] are enhanced by the inclusion of more values of $m_{Q}$, by a further analysis of the $O\left(1 / m_{Q}\right)$ terms that contribute to $f_{P}$, and by the use of the tad-

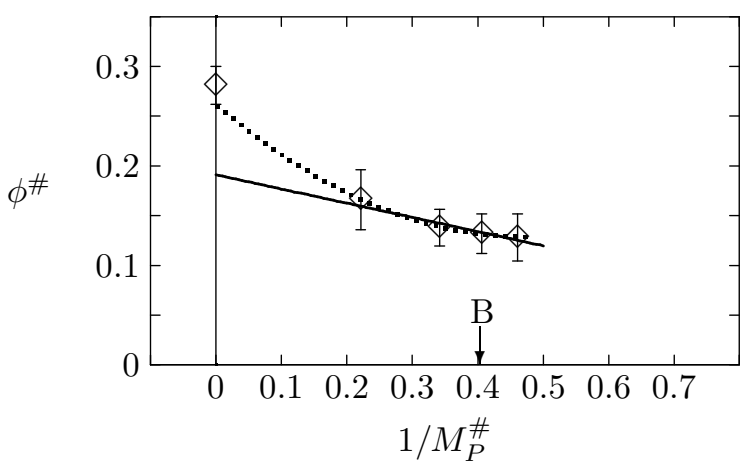

Figure 4. Quenched SGO results for $\phi^{\#}$ as a function of the inverse heavy-light meson mass $1 / M_{P} \#$ at the chiral limit [31]. The results of a linear and quadratic fit in $1 / M_{P}^{\#}=1 /\left(M_{P} a\right)$ to the NRQCD data points is also shown.

pole improved Clover action for the light quarks. Again the preliminary values for the renormalisation constant were used [39]. For the Wilson data only two values of the light quark mass were available meaning that chiral extrapolations were not robust. The Clover data has three light quark values.

In fig.5 a plot of $Z^{\text {Ren }} \phi^{\#}=f_{P} \sqrt{M_{P}} a^{3 / 2}$ (see 
eq.(1D) versus $1 / M_{P}^{\#}$ is displayed. In this graph both Wilson (at $\left.\kappa_{q}=0.1585\right)$ and Clover $\left(\kappa_{q}=\right.$ $0.1385)$ data are plotted. The $\kappa_{q}$ values are chosen so that the corresponding pion masses agree. Note that in order to make the comparison, the appropriate renormalisation factor for both actions has been included. The curves are cubic fits in $1 / M_{P}^{\#}$ to the NRQCD data only. The static points are also plotted, but not included in the fits.

There are three important points to be made:

- The NRQCD data points smoothly extrapolate to the static point. Thus the inclusion of more data points with larger $m_{Q}$ seems to have resolved the discrepancy that appeared earlier [40].

- The slope of $\phi$ against $1 / M_{P}$ is apparently increasing as $M_{P} \rightarrow \infty$. This signifies the presence of terms of order $1 / m_{Q}^{2}$. However, the calculation is correct only to $O\left(1 / m_{Q}\right)$. Therefore, strictly speaking, any non-constant behaviour of this slope at finite $m_{Q}$ is not a real prediction of these calculations. In practice though, the behaviour seen in fig 5 matches well with data from conventional simulations at around the D-meson (see sec. 5). So presumably it true is that the present simulation does not suffer from neglecting terms of order $1 / \mathrm{m}_{Q}^{2}$. Note also that from the figure there is a $30 \%$ $1 / M_{P}$ correction to $f_{B}$ compared with the static value.

- The Wilson data is statistically lower than the Clover data. The obvious explanation is that this is a symptom of $O(a)$ effects spoiling the Wilson data 26.). (These systematics could enter either in $\phi^{\#}$ or in $a^{-1}$.) It could also be that the one-loop perturbative calculation of $Z^{\text {Ren }}$ is inadequate, and that a non-perturbatively defined $Z^{\text {Ren }}$ would remove the disagreement.

In the SGO analysis the contributions of the three $O\left(1 / m_{Q}\right)$ terms to $\phi$ were extracted. Expressing these as $c_{i} / M_{P}$ they found that

$\left|c_{D^{2}}\right|>\left|c_{\sigma . D}\right|>>\left|c_{\sigma . B}\right|$

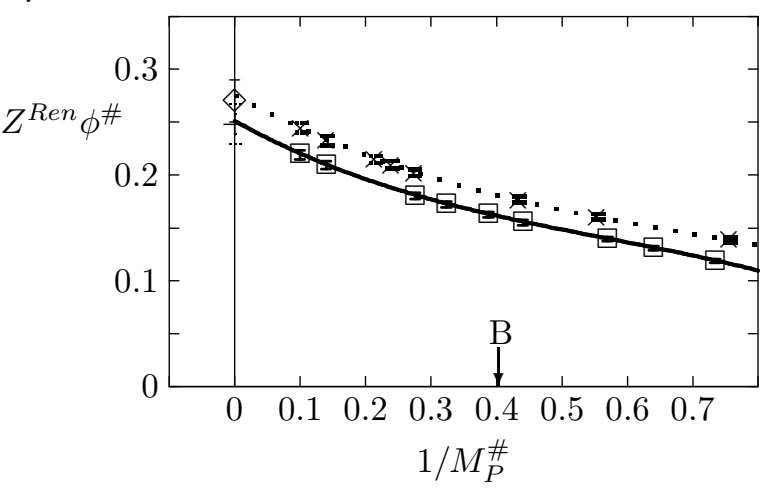

Figure 5. Dynamical SGO results for $Z^{R e n} \phi^{\#}$ as a function of the inverse heavy-light meson mass $1 / M_{P}^{\#}[27]$. The upper set of data uses the Clover action for the light quark (with $\kappa_{q}=0.1385$ ), and the lower set uses the Wilson action (with $\left.\kappa_{q}=0.1585\right)$. The results of cubic fits in $1 / M_{P}$ to the NRQCD data points are also shown.

That the hyperfine component, $c_{\sigma . B}$ is smallest fits our expectations since this term breaks both the spin and flavour symmetry present in the $m_{Q}=\infty$ limit 41,27 .

Performing a chiral extrapolation and using a nominal value of $a^{-1}=2 \mathrm{GeV}$, one obtains the values for $f_{B}$ and $f_{B} / f_{B_{S}}$ which appear in table 3, 27. This (rough) $f_{B}$ value is in nice agreement with that obtained by the quenched NRQCD analysis of [31] implying that the effects of dynamical quarks are at, or below, the level of statistics. The difference between the two actions for the chiral ratio is unexpected since systematic effects such as $O(a)$ are expected to cancel in this ratio.

\section{Conventional Results}

The "conventional" formalism of the heavyquark propagator is simply that obtained from the lattice version of the Dirac equation, i.e. the same lattice action as for the light degrees of freedom. However, potential problems exist when $m_{Q} a \gtrsim 1$. There are two approaches to reduce discretisation effects. The first is to use an im- 
proved action, such as the Clover action 泊, which has all terms $O\left(m_{Q} a\right)$ explicity removed [42]. The second is to use the "Fermilab" formulation of 5 8. These two choices are not mutually exclusive, and while all groups use the second technique, one group [43] uses both.

Table 1 summarise the data to appear recently with the references in the second row. In the following subsections a brief review of each of the four groups' work is presented.

\subsection{MILC Results}

The MILC collaboration's results [28] distinguish themselves because they are the only simulations in the conventional approach that (i) have dynamical fermion runs, (ii) have a static point to help constrain their fits in $1 / M_{P}$, and (iii) use the hopping parameter expansion to give them a wide range of $\kappa_{Q}$ values at very little cost 444. They have also studied finite volume effects, and performed a continuum extrapolation. (This last point will be discussed more later.)

In fig.6 the quantity $\phi$ is plotted against $1 / M_{P}$, both in physical units. The curve shown is a fit to the points marked with a cross (i.e. in this case, points with $M_{P}<M_{D}$ ). By varying the fitting window in $M_{P}$, an estimate is obtained of the error due to the $m_{Q} a$ effects which escape the Fermilab redefinitions. Another estimate of this error would be to fit the standard Wilson data (without the Fermilab redefinitions) for, say, $M_{P}<M_{D}$, and compare it with the results from Fermilab data for the same $M_{P}$ range.

Fig.7 shows the continuum extrapolation of $f_{B}$ with $f_{\pi}$ used to set the scale. Both quenched and dynamical data are shown. The line shown is a linear fit to all the quenched data (i.e. $5.7 \leq$ $\beta \leq 6.5)$. It is probable that this procedure suffers from the same problem discussed in sec. 3.3 , where static data for $\beta \lesssim 6.0$ was found not to scale, within statistical errors. If this scenario is correct, then including the data with $\beta \lesssim 6.0$ in a linear fit skews the continuum extrapolation downwards. Fig.7 seems to confirm this assertion, though the effect is small. This would explain at least part of the difference between the MILC results and the other data shown in table 1 .

The dynamical data points in the figure show a

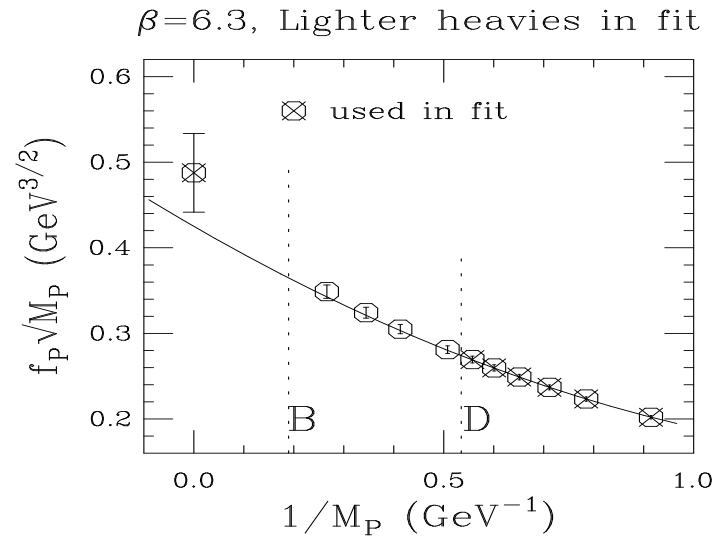

Figure 6. MILC results for $\phi$ as a function of $1 / M_{P}[28]$. The data points used in this particular fit are denoted with a cross (see text).

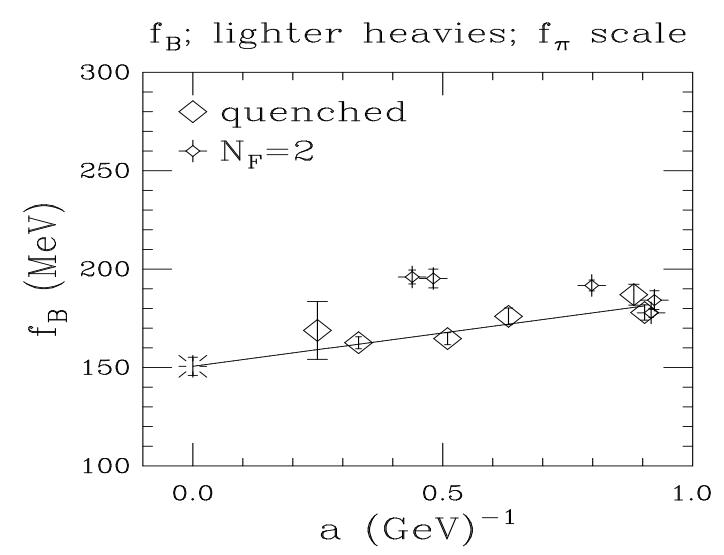

Figure 7. MILC results for $f_{B}$ as a function of lattice spacing $a$ [28]. The fit is to the quenched points only (see text).

clear discrepancy at around $a=0.5 \mathrm{GeV}^{-1}$ compared with the quenched data. This can be used as an estimate of the quenching errors which appears to be considerable at this stage [28].

The results of the MILC collaboration's work is displayed in table 4 . The three errors stated are: (i) statistical, (ii) various systematic, and (iii) quenching. The systematic errors are a combination of the uncertainties in the various fits, the $m_{Q} a$ effects, finite volume and finite $a$ errors. 
Table 4

Summary of recent results using the conventional approach. 'Q' signifies Quenched simulation, 'D' Dynamical. See text for detailed comments, and a description of the various errors. All numbers should be considered preliminary.

\begin{tabular}{|c|c|c|c|c|}
\hline Collaboration & $\begin{array}{l}\text { MILC } \\
28\end{array}$ & $\begin{array}{l}\text { LANL - Ohio } \\
\text { Washington }\end{array}$ & $\begin{array}{c}\text { JLQCD } \\
\\
46 \mid\end{array}$ & $\begin{array}{c}\text { FNAL } \\
43\end{array}$ \\
\hline \multicolumn{5}{|c|}{ Lattice Parameters } \\
\hline$n_{F}$ & $0 \& 2$ & 0 & 0 & 0 \\
\hline$\beta$ & $\begin{array}{l}5.7 \rightarrow 6.5 \mathrm{Q} \\
5.4 \rightarrow 5.7 \mathrm{D}\end{array}$ & 6.0 & $6.1,6.3$ & 5.9 \\
\hline $\begin{array}{l}\text { Volume } \\
N_{c f g s} \\
S_{q} \\
S_{Q}\end{array}$ & $\begin{array}{c}\leq 32^{3} \times 100 \\
\sim 100 \\
\text { Wilson } \\
\text { Fermilab-Wilson } \\
\quad \text { \& Static }\end{array}$ & $\begin{array}{c}32^{3} \times 64 \\
170 \\
\text { Wilson } \\
\text { Fermilab-Wilson }\end{array}$ & $\begin{array}{c}\leq 32^{3} \times 80 \\
\sim 100 \\
\text { Wilson } \\
\text { Fermilab-Wilson }\end{array}$ & $\begin{array}{c}16^{3} \times 32 \\
100 \\
\text { Clover } \\
\text { Fermilab-Clover }\end{array}$ \\
\hline $\begin{array}{l}\text { Results } \\
f_{D}[\mathrm{MeV}] \\
f_{D_{s}} \\
f_{B}[\mathrm{MeV}] \\
f_{B_{S}} \\
\end{array}$ & $\begin{array}{c}182 \pm 3(9)(22) \\
198 \pm 5(10)(19) \\
151 \pm 5(16)(26) \\
169 \pm 7(14)(29)\end{array}$ & $\begin{array}{c}229 \pm 7(-)(+12)\left({ }_{-14}^{+18}\right)(7) \\
260 \pm 4(-5)(+15)\left({ }_{-20}^{+24}\right)(8)\end{array}$ & $\begin{array}{c}214 \pm 10(25) \\
248 \pm 7(29) \\
221 \pm 15(26) \\
244 \pm 8(28) \\
\end{array}$ & $\begin{array}{c}220_{-5}^{+4} \\
\left(239_{-4}^{+3}\right) \\
\left.188_{-4}^{+6}\right) \\
\left(207_{-2}^{+3}\right) \\
\end{array}$ \\
\hline
\end{tabular}

\subsection{LANL-Ohio-Washington Results}

The LANL-Ohio-Washington collaboration have new results using the Fermilab Wilson formulation [45]. Since they have no static point, and are working at a moderate $\beta$ value, no estimate of $f_{B}$ was given. Of particular interest is their comprehensive study of the systematics involved in setting the strange and charm quark masses, and in the definition of $Z^{\text {Ren }}$. This is outlined below.

- The hopping parameter corresponding to the strange quark mass, $\kappa_{s}$, is defined in three different ways: by fixing $M_{K}^{2} / M_{\pi}^{2}$; $M_{K}^{*} / M_{\rho}$; and $M_{\phi} / M_{\rho}$ to be equal to their physical values. These estimates correspond to a surprisingly large $\sim 20 \%$ spread in the values of the strange quark mass.

- The charm quark is set by fixing the mass of a meson containing a c-quark to its experimental value. The lattice estimate of these heavy-light meson masses is defined using either the pole mass (obtained from the exponential decay of the correlation functions), or by the lattice dispersion relation.
- A careful study of the systematic effects entering the definition of $Z^{R e n}$ was undertaken. Here different values of the LepageMackenzie $q^{*}$ were used [23], and two definitions $\left(u_{0}=1 / 8 \kappa_{c}\right.$ and $\left.<U_{\text {plaq }}>^{1 / 4}\right)$ were used to redefine the coupling.

The spread of decay constant values using each of these methods gives estimates of the corresponding systematic effects. The final results for $f_{D}$ and $f_{D_{s}}$ are shown in table 1 . The five errors listed are due to: (i) statistics, (ii) strange quark determination, (iii) charm quark determination, (iv) $Z^{R e n}$, (v) setting the scale. The importance of this work is that it shows that each of the three errors outlined above are at least of the same order as the statistical and scale errors (which are normally assumed to be the dominant errors).

\subsection{JLQCD Results}

A status report of the ongoing analysis by the JLQCD collaboration was presented at this conference [46]. They have data at two $\beta$ values and use a quadratic fit in $1 / M_{P}$ to determine their values for $f_{B}, f_{D}$ etc. Their results, taken from the $\beta=6.3$ dataset are given in table 1 . The $\rho$ 
mass was used to set the scale. The first quoted error is statistical, and the second is due to the scale. More accurate results will be obtained from this collaboration in the near future.

\subsection{FNAL Results}

The FNAL group are simulating the Clover action in the Fermilab formalism at $\beta=5.9$ [43]. They have preliminary results which are shown in table 4 . (Statistical errors only are shown.) The strong point of this work is that it uses the Clover action (i.e. complete with the rotations of the quark fields) and that therefore the results should be free of $O\left(m_{Q} a\right)$ effects. Values for the decay constants in the table use $f_{\pi}$ to set the scale. $f_{D_{s}}$ and $f_{B_{S}}$ are quoted in parentheses, since the strange quark value used in their determination is nominal. The FNAL group plan to simulate at other $\beta$ values to check the scaling of these quantities.

\section{Conclusions}

One of the main themes of this review is that systematic effects now clearly dominate statistical errors in lattice calculations of $f_{P}$. In many ways this is a very desirable situation since it allows their careful callibration. I list the main systematic uncertainties and their possible solutions.

- $Z^{\text {Ren }}$ varies by as much as $\sim 30 \%$ (see secs. 3.2 and 5.2). This variation can be significantly improved by using the clover action. The non-perturbative value of $Z^{\text {Ren }}$ will clear up this uncertainty [24].

- The continuum limit of $f_{P}$ can be studied by an $a \rightarrow 0$ extrapolation. In the static case, only data with $\beta \lesssim 6.0$ should be included in this fit (for present levels of statistics, see sec. 3.3), otherwise the $a=0$ value will be biased downwards. Presumably a remnant of this effect survives in the NRQCD and conventional cases. Clearly $O(a)$ effects should be reduced by the use of the clover action.

- Dynamical $\left(n_{F}=2\right)$ results seem to produce higher values for $f_{P}$ for both the NRQCD (sec. 4.2) and conventional cases (sec. 5.1). Whether this effect is invariant under further investigation will be a main source of research over the next couple of years. A more subtle question is the extent to which $n_{F}=2$ data truly reflects the real $n_{F} \approx 3$ world 47,48 .

- The choice of physical quantity to set $a$ impacts upon the final value of $f_{P}$. Again, short-term, this should be fed into the systematic uncertainties, but longer-term this should be understood in terms of quenching and/or $O(a)$ errors.

- The chiral extrapolation of methods which use a fixed smearing for all light quark values should be questioned (see 3.5). This is not a large effect for $f_{P}$ but can become significant for chiral ratios/differences such as $f_{B_{S}} / f_{B}$ and $M_{B_{S}}-M_{B}$.

- Setting the hopping parameters corresponding to the physical strange and charm quarks also introduces an error which should be included (see sec. 5.2)

The wide range of $M_{P}$ values presently covered by the three methods allow an important consistency test to be made: Do the different approaches agree with eachother? Studying the $f_{B}$ values in tables 2.3 and 4. together with the $\phi$ versus $1 / M_{P}$ plots suggests that they do. Note that there is still a fair degree of 'slop' in the data due to the fact that different groups have used different definitions of $Z^{R e n}$ and chosen different quantities to set the scale. Once this is tightened up, this consistency test can be made more definitive (e.g. by using all three methods to determine $f_{P}$ on the same configurations).

Using the data in the tables I estimate the following "global" lattice averages: $f_{D} \approx f_{B}=$ $200 \mathrm{MeV} \pm 20 \%$. The error bar includes my estimate of all uncertainties.

\section{Acknowledgements}

I wish to thank my collaborators M. Crisafulli, V. Lubicz, G. Martinelli, F. Rapuano and A. Vladikas for many useful discussions over the last 
several stimulating years in Rome. Supported from the EU Human Capital and Mobility Programme, Grant ERBCHBICT 941462 is much appreciated.

\section{REFERENCES}

1. J. Rosner, J.Phys. G 18 (1992) 1575

2. G.P. Lepage et al, Phys. Rev. D 46 (1992) 4052, and references therein

3. B. Thacker and G.P. Lepage, Phys. Rev. D 43 (1991) 196

4. B. Sheikholeslami and R. Wohlert, Nucl. Phys. B 259 (1985) 572

5. A. El-Khadra, Nucl. Phys. B (Proc. Suppl.) 26 (1992) 372

6. P.B. Mackenzie, Nucl. Phys. B (Proc. Suppl.) 30 (1993) 35

7. A. Kronfeld, Nucl. Phys. B (Proc. Suppl.) 30 (1993) 445

8. A. El-Khadra, A. Kronfeld and P.B. Mackenzie, in preparation

9. E. Eichten, Nucl. Phys. B (Proc. Suppl.) 4 (1988) 170

10. E. Eichten and F. Feinberg, Phys. Rev. D 23 (1981) 2724

11. G.P. Lepage, Nucl. Phys. B (Proc. Suppl.) 26 (1992) 45, A. Duncan et al, ibid 391

12. Ph. Boucaud et al, Phys. Lett. B 220 (1989) 219

13. C.R.Allton, C.T.Sachrajda, V.Lubicz, L.Maiani and G.Martinelli, Nucl. Phys. B 349 (1991) 598

14. A. Duncan, E. Eichten, J. Flynn, B. Hill, G. Hockney and H. Thacker, Phys. Rev. D 51 (1995) 5101

15. S. Güsken, Nucl. Phys. B (Proc. Suppl.) 17 (1990) 361

16. C. Alexandrou et al, Nucl. Phys. B414 (1994) 815

17. T. Draper and C. McNeile, Nucl. Phys. B (Proc. Suppl.) 34 (1994) 453

18. A. Kronfeld, Nucl. Phys. B (Proc. Suppl.) 17 (1990) 313

19. M. Lüscher and U. Wolff, Nucl. Phys. B339 (1990) 222

20. E. Eichten, G. Hockney and H.B. Thacker, Nucl. Phys. B (Proc. Suppl.) 17 (1990) 529
21. E. Eichten and B. Hill, Phys. Lett. B 240 (1990) 193, Ph. Boucaud, C.L. Lin and O. Pène, Phys. Rev. D 40 (1989) 1529

22. A. Borrelli and C. Pittori, Nucl. Phys. B 385 (1992) 502, O.F. Hernández and B.R. Hill, Phys. Lett. B 289 (1992) 417

23. G.P. Lepage and P.B. Mackenzie, Phys. Rev. D 48 (1993) 2250

24. M. Talevi, these proceedings

25. C.W. Bernard, Nucl. Phys. B (Proc. Suppl.) 34 (1994) 47

26. C.R. Allton Nucl.Phys. B 437 (1995) 641

27. S. Collins, these proceedings

28. C. Bernard, these proceedings

29. D.G. Richards, these proceedings

30. UKQCD Collab., A.K. Ewing et al, heplat/9508030

31. A. Ali Khan, these proceedings

32. C.R. Allton, M. Crisafulli, V. Lubicz, G. Martinelli, F. Rapuano and A. Vladikas, in preparation

33. APE Collab., C.R. Allton et al, Nucl. Phys. B413 (1994) 461

34. APE Collab., C.R. Allton et al, Phys. Lett. B326 (1994) 295

35. APE Collab., C.R. Allton et al, Nucl. Phys. B (Proc. Suppl.) 42 (1995) 385

36. A. Ali Khan, Nucl. Phys. B (Proc. Suppl.) 42 (1995) 382

37. J.H. Sloan, Nucl. Phys. B (Proc. Suppl.) 42 (1995) 171

38. T. Draper, these proceedings

39. C. Morningstar, J. Shigemitsu et al, in preparation

40. S. Collins, Nucl. Phys. B (Proc. Suppl.) 42 (1995) 394

41. M. Neubert, Phys. Rev. D 46 (1992) 1076

42. G. Heatlie et al, Nucl. Phys. B 352 (1991) 266

43. J. Simone et al., in preparation

44. D.S. Henty and R.D. Kenway, Phys. Lett. B 289 (1992) 109

45. T. Bhattacharya, R. Gupta, G. Kilcup and S. Sharpe, LAUR-95-2355

46. S. Hashimoto, these proceedings

47. S. Sharpe, these proceedings

48. M.J. Booth, Phys. Rev. D 51 (1995) 2338 and hep-ph/9412228 